Rev. Biol. Trop. 52 (3): 745-756, 2004

www.ucr.ac.cr www.ots.ac.cr www.ots.duke.edu

\title{
Percepción de la biotecnología en estudiantes universitarios de Costa Rica
}

\author{
Marta Valdez ${ }^{1}$, Iris Rodríguez ${ }^{1} \&$ Ana Sittenfeld ${ }^{2}$ \\ 1 Escuela de Biología, Universidad de Costa Rica, Ciudad Universitaria Rodrigo Facio, San José, Costa Rica; \\ mvaldez@biologia.ucr.ac.cr. \\ 2 Centro de Investigación en Biología Celular y Molecular, Universidad de Costa Rica, Ciudad Universitaria Rodrigo \\ Facio, San José, Costa Rica.
}

Recibido 14-VII-2004. Corregido 02-IX-2004. Aceptado 02-IX-2004.

\begin{abstract}
Perception about biotechnology in university students in Costa Rica. A survey was carried out to determine the perception and knowledge about biotechnology and genetically modified organisms (GMOs) in a sample $(n=750)$ of university students from three public universities in Costa Rica: Universidad de Costa Rica, Universidad Nacional and Instituto Tecnológico de Costa Rica. The study revealed that $88 \%$ of the students showed a satisfactory level of knowledge about modern biotechnology and 79\% of them reported a favorable opinion and good acceptance of this technology. Students would accept some risks associated to biotechnology if it represents an improvement to the competitiveness of Costa Rica. Some differences were detected in the opinions from students of the three universities that can be associated to the area of study. Students from social disciplines showed a higher percentage of negative acceptances to biotechnology and GMOs when their opinions were compared with those of students from life sciences and technologies. Rev. Biol. Trop. 52(3): 745-756. Epub 2004 Dic 15.
\end{abstract}

Key words: Costa Rica, biotechnology, university students, genetically modified organisms, GMOs, public perception.

Palabras clave: Costa Rica, biotecnología, estudiantes universitarios, organismos modificados geneticamente, OMGs, percepción pública.

La genética ha avanzado considerablemente en las últimas décadas, debido a una serie de descubrimientos que se inician con la identificación del ADN como el material hereditario, la elucidación de la estructura en doble hélice de la molécula de ADN por Watson y Crick en 1953, la identificación del código genético, la habilidad para aislar genes, la aplicación de las tecnologías de ADN recombinante, la producción de organismos modificados genéticamente (OMGs) y más recientemente el desarrollo de la genómica y la bioinformática. Estos avances han permitido una revolución en las ciencias biológicas, que han acelerado no sólo el entendimiento de los procesos celulares, sino que han proporcionado las herramientas a la biotecnología moderna para la producción de bienes y servicios. Así, se considera que estas disciplinas han tenido un gran impacto en las áreas de la salud, industria y agricultura, con profundas repercusiones en el mejoramiento de la calidad de vida (Sasson 1985, BIO 2002, Albertazzi 2004).

La ingeniería genética aplicada a los cultivos de importancia económica contribuye al mejoramiento genético convencional, con la generación de especies con características novedosas como resistencia a herbicidas e insectos (James 2003). A nivel mundial, el área global de cultivos transgénicos aumentó considerablemente, de 1.7 millones de hectáreas en 1996 a 67.7 en el 2002. Ese año, unos 6 millones de agricultores cultivaban más de 70 variedades de plantas transgénicas, en 18 países, 
especialmente en los Estados Unidos, Argentina, China y Canadá. Más de un cuarto de esa área se sembró en varios países en vías de desarrollo, que incluyen a Sudáfrica, Honduras, México, Colombia, Brasil, India e Indonesia. $\mathrm{El}$ incremento en la superficie sembrada se atribuye a que esas variedades ofrecen mejores rendimientos, resistencia a factores abióticos, enfermedades, pestes y mejoras en las características nutricionales (James 2003). Sin embargo, como toda tecnología, su aplicación tiene ventajas y desventajas potenciales, dependiendo de su adecuada utilización (Priest 2000).

La producción de OMGs en agricultura ha generado diversidad de opiniones en la comunidad mundial, en cuanto a su uso y aceptación (Priest 2000). En este contexto, la percepción pública hacia la biotecnología y particularmente hacia la inclusión de OMGs en los alimentos, varía en los diferentes países desarrollados, y parece ser más negativa en los países europeos que en los Estados Unidos (Gaskell et al. 2000, 2003, Priest 2000). El debate en torno a este tema no ha estado ausente en los países en desarrollo. No obstante, la información es escasa y no existen suficientes estudios que permitan determinar los niveles de aceptación de los OMGs en la población general, así como las actitudes hacia la biotecnología en estos países (Aerni 2001, Huete-Pérez y Orozco-González 2001). Según Solleiro y Castañón (2002), en América Latina, los niveles de percepción pública negativa sobre los alimentos y productos transgénicos son bastante altos: $45 \%$ en Brasil, $50 \%$ en México, $73 \%$ en Chile. Lo atribuyen a la intensa campaña de divulgación contra la biotecnología, llevada a cabo por grupos ambientalistas radicales. Sólo en Cuba y en Uruguay se encontró un apoyo explícito y amplio de los sectores gubernamentales, así como niveles altos de apoyo popular hacia la biotecnología moderna.

En Costa Rica, los centros de investigación en las universidades estatales han desarrollado programas en biotecnología agrícola desde hace unos 20 años, apoyados por los sectores gubernamentales responsables del desarrollo de la ciencia y la tecnología en el país.
Esto ha favorecido el establecimiento de compañías privadas locales, dedicadas a la biotecnología de plantas, particularmente a sistemas de propagación in vitro. El desarrollo de programas de mejoramiento genético asistido con técnicas biotecnológicas como la ingeniería genética, se realiza en los centros de investigación pública (Sittenfeld y Espinoza 2002). Así, la tecnología transgénica se desarrolla desde 1990, cuando el Programa de Biotecnología del Arroz del Centro de Biología Celular y Molecular (CIBCM) de la Universidad de Costa Rica, inició investigaciones para generar arroz transgénico con resistencia al "virus de la hoja blanca del arroz" (RHBV). Este programa tiene un enfoque multidisciplinario e integral que incluye: evaluación de flujo génico del arroz transgénico a especies silvestres y malezas relacionadas, bioseguridad alimenticia y ambiental, así como aspectos de propiedad intelectual y transferencia de la tecnología a los agricultores (Espinoza et al. 2003). Otros proyectos de investigación en el área de cultivos transgénicos, que se encuentran a nivel experimental confinado (laboratorio e invernadero), y que ha desarrollado el sector público costarricense, incluyen la transformación de variedades de maíz, y del tubérculo "Tiquisque" (Xanthosoma sagittifolium) en la Universidad de Costa Rica. El Centro Agronómico Tropical de Investigación y Enseñanza (CATIE) realiza investigaciones sobre la transformación de variedades de plátanos (Musa sp.), para conferir resistencia a la enfermedad fúngica "Sigatoka negra".

Una encuesta a escala nacional, realizada entre mayo y junio del 2001, para determinar el grado de conocimiento y la percepción de los OMGs en Costa Rica, reveló en general, una percepción pública positiva. Sin embargo, más del 50\% de los encuestados señalaron no haber escuchado o leído sobre cultivos modificados genéticamente. Así mismo, se encontró una relación entre la aceptación de los OMGs y el nivel educativo de los encuestados (Garita-Bonilla 2001, Sittenfeld y Espinoza 2002).

El objetivo de la presente investigación fue examinar la percepción de la biotecnología y el grado de conocimiento en dicha materia 
en una muestra poblacional con alto grado educativo, correspondiente a estudiantes de tres universidades públicas de Costa Rica.

\section{MATERIALES Y MÉTODOS}

Se seleccionaron tres universidades públicas de Costa Rica: Universidad de Costa Rica (UCR), Universidad Nacional (UNA) e Instituto Tecnológico de Costa Rica (ITCR). Se aplicó una encuesta a una muestra representativa de los estudiantes de esas instituciones $(\mathrm{n}=250 \mathrm{de}$ cada una), que fue tomada al azar. El tamaño de la muestra fue seleccionado de acuerdo con el método de muestreo de proporciones y porcentajes de Cochran (1963) y de Gómez-Barrantes (1998). El nivel de confianza de los resultados fue de $90 \%$ y el margen de error de $\pm 5 \%$.

A cada encuestado se le tomaron datos de edad, género y carrera de estudio. El cuestionario se aplicó en forma personal a un total de 750 estudiantes con una edad promedio de 20 años, durante los meses de mayo a octubre de 2001 en lugares de alta afluencia de estudiantes (zonas aledañas a bibliotecas y cafeterías). Varias de las preguntas para el presente estudio, fueron adaptadas para el contexto nacional a partir del Eurobarómetro (Durant 2000, Gaskell et al. 2000). La encuesta consistió en cuatro partes. La primera trató de estimar la opinión sobre el impacto de 6 nuevas tecnologías (Biotecnología, Computación, Energía Solar, Exploración Espacial, Nuevos Materiales, y Telecomunicaciones) en la calidad de vida en los próximos 20 años. El análisis de las respuestas sobre la biotecnología, se realizó en forma independiente para las muestras de estudiantes de cada universidad. La segunda parte, de 5 preguntas, permitió estimar el nivel de conocimiento sobre la biotecnología y la ingeniería genética en la población muestreada. Los encuestados opinaron si estaban o no de acuerdo con cada uno de los 5 enunciados sobre la materia, que podían ser correctos o incorrectos. Cada uno de los 5 enunciados, se presenta en el Cuadro 1 .

\section{CUADRO 1}

Porcentaje (\%) de entrevistados de cada una de las universidades públicas de Costa Rica consideradas en el estudio*, que respondieron si estaban de acuerdo o en descuerdo con cinco enunciados (1 a 5), que pueden ser o no correctos, sobre biotecnología e ingeniería genética

\section{Enunciados}

1. Los tomates comunes no contienen genes, mientras que los modificados genéticamente si los tienen.

2. La clonación de organismos vivos produce una descendencia idéntica al progenitor.

3. Los genes de una persona se pueden modificar si come una fruta transgénica.

4. La levadura para producir cerveza consiste en organismos vivos.

5. Los animales genéticamente modificados siempre son más grandes que los no modificados.

\section{Respuesta}$$
\text { De }
$$

De acuerdo
En desacuerdo
No responde

UCR (\%)

UNA (\%)

ITCR (\%)

Promedio

De acuerdo
En desacuerdo
No responde

12
87.2
0.8

\section{6}

68.8

3.6

15.6

83.6

0.8

18.4

79.8

69.2
30.8
0

De acuerdo
En desacuerdo
No responde

\section{8}

89.2

0

60
39.6
0.4

53.6

45.6

0.8

61

38.6

0.4

De acuerdo
En desacuerdo
No responde

\section{8}

12.4

0.8

$$
23.2
$$

75.2

12

86.4

15.3

83.6

$\begin{array}{lll}1.6 & 1.6 & 1.1\end{array}$

De acuerdo

En desacuerdo

11.2

No responde

\section{2}

78.4

80.1

21.6

3.2 
Para estimar el grado de conocimiento general en relación con el conjunto de esas declaraciones, se tomaron en cuenta los 5 enunciados para cada encuestado. Si la persona acertó 4 o 5 respuestas, se estimó que tenía buen conocimiento sobre el tema y se clasificó como "Bastante Bueno"; si tenía 3 respuestas correctas se clasificó como "Bueno" y si acertó 0,1 ó 2 respuestas fue clasificado como "Insuficiente". En la tercera parte, se interrogó sobre qué instituciones u organizaciones, creían los encuestados que debían ser las entidades encargadas de los aspectos regulatorios de las actividades biotecnológicas. Entre las entidades presentadas a consideración se incluyeron: la Asamblea Legislativa, comités de ética, organizaciones científicas, organismos públicos del Estado, y organizaciones internacionales. En la cuarta y última parte, se trató de estimar el grado de aceptación de los encuestados sobre los organismos y alimentos transgénicos, así como los riesgos asociados con la biotecnología moderna, por medio de tres preguntas o enunciados. Cada uno de ellos, se presenta en el Cuadro 3. Los encuestados opinaron si estaban o no de acuerdo con cada una de esas afirmaciones. Para estimar el grado de aceptación general en relación con esas declaraciones, se tomaron en cuenta las 3 respuestas para cada encuestado. Si la persona estuvo de acuerdo con los tres enunciados se consideró como "Bastante buena", con dos enunciados como "Buena", y con uno o ninguno de ellos, como "No aceptan".

Para relacionar el conocimiento y la percepción sobre la biotecnología y los OMGs con la carrera universitaria de estudio de los encuestados, se realizó una agrupación de estos de acuerdo con la carrera cursada. Estas, se clasificaron en cinco áreas de estudio (Artes y Letras, Ciencias Básicas, Ciencias Sociales, Ingenierías y Ciencias de la Salud).

La información obtenida se introdujo en una base de datos en el programa Access de Microsoft ${ }^{\mathrm{TM}}$, lo cual permitió su análisis ulterior. A los resultados se les aplicó la prueba estadística chi-cuadrado $\left(X^{2}\right)$ para determinar si las muestras eran estadísticamente homogéneas. Para las preguntas donde las respuestas fueron homogéneas, se realizó el cálculo estadístico, considerando la totalidad de la población encuestada. Posteriormente se elaboraron cuadros y figuras para su análisis e interpretación.

\section{RESULTADOS}

La mayoría de los encuestados consideraron que las nuevas tecnologías en general, mejorarán la calidad de vida de la humanidad en los próximos 20 años (Fig. 1). Las tecnologías relacionadas con la energía solar y con las computadoras fueron consideradas como las de mayor beneficio. Con respecto al impacto de la biotecnología en la calidad de vida, se observa que la mayoría opina que esta tecnología podría mejorarla $(83.4 \%$, Fig. 1$)$. En relación con la opinión sobre la biotecnología, al analizar las respuestas para la muestra poblacional de cada universidad (Fig. 2), no se encontró diferencia significativa entre los estudiantes de las tres universidades $\left(X^{2} \mathrm{p}>\right.$ $0.5)$. La mayoría de ellos consideró que esa tecnología podría ser bastante provechosa para la sociedad en el futuro.

Los resultados de la segunda parte de la encuesta para cada una de las universidades, se presentan en el Cuadro 1. En relación con el primer enunciado: "Los tomates convencionales no contienen genes, mientras que los modificados genéticamente si los tienen", la mayoría de los entrevistados de las tres universidades contestaron acertadamente la opción "En desacuerdo". Para el enunciado 2: "La clonación de organismos vivos produce una descendencia idéntica al progenitor", la respuesta correcta era la opción "De acuerdo", y al agrupar los resultados para las tres universidades, se observa que un $61 \%$ respondieron de manera acertada. El enunciado 3: "Los genes de una persona se pueden modificar si come una fruta transgénica" se refiere al conocimiento de la influencia que podrían tener los genes foráneos sobre la estabilidad genética de las personas. La opción de respuesta correcta a esta pregunta es "En desacuerdo", para cual un $83.6 \%$ de los encuestados en general (Cuadro 


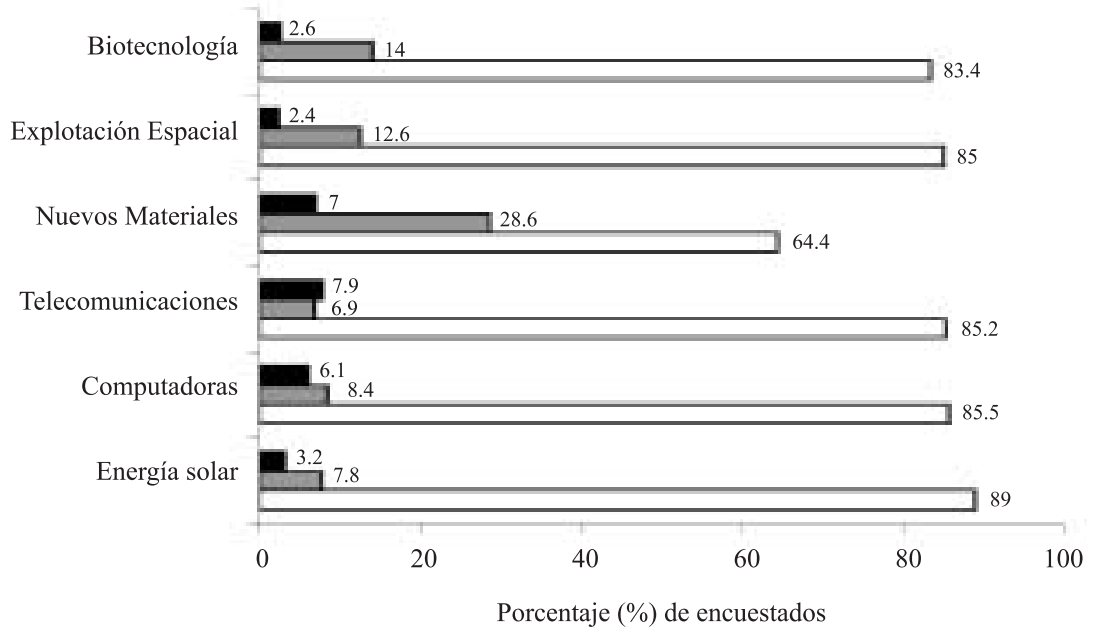

Fig. 1. Opinión de estudiantes de 3 universidades públicas de Costa Rica*, sobre el impacto de las nuevas tecnologías en el mejoramiento de la calidad de vida en los próximos 20 años. Nota (*) Universidad de Costa Rica (UCR), Universidad Nacional (UNA) e Instituto Tecnológico de Costa Rica (ITCR). $\square$ Mejorará $\square$ No mejorará $\square$ No saben.

Fig. 1. Opinion from students of 3 Costa Rican public universities*, on the impact of new technologies to improve quality of life in the next 20 years. Note (*) Universidad de Costa Rica (UCR), Universidad Nacional (UNA) and Instituto Tecnológico de Costa Rica (ITCR). $\square$ Will improve $\square$ Will not improve $\square$ Unknown.

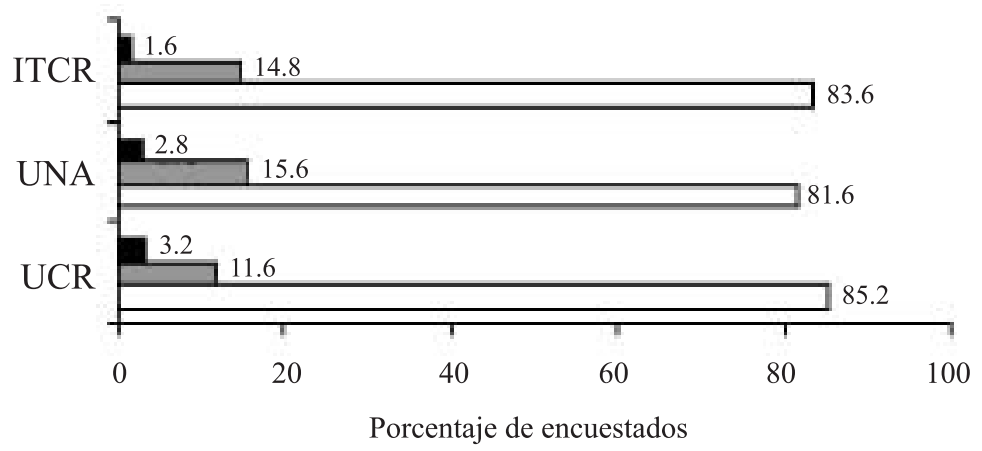

Fig. 2. Opinión de estudiantes de 3 universidades públicas de Costa Rica*, sobre el impacto de la biotecnología en el mejoramiento de la calidad de vida en los próximos 20 años. Nota (*) Universidad de Costa Rica (UCR), Universidad Nacional (UNA) e Instituto Tecnológico de Costa Rica (ITCR). $\square$ Mejorará $\square$ No mejorará $\square$ No saben.

Fig. 2. Opinion from students of 3 Costa Rican public universities*, on the impact of biotechnology to improve quality of life in the next 20 years. Note $\left(^{*}\right)$ Universidad de Costa Rica (UCR), Universidad Nacional (UNA) and Instituto Tecnológico de Costa Rica (ITCR). $\square$ Will improve $\square$ Will not improve $\square$ Unknown. 
1), respondieron acertadamente a esta afirmación. El cuarto enunciado trata sobre el conocimiento básico del proceso biotecnológico tradicional de la fermentación alcohólica por parte de levaduras, que representa una de las primeras actividades biotecnológicas desarrolladas por el hombre. La opción de respuesta correcta es "De acuerdo", para la cual un $80.1 \%$ del promedio de los encuestados de las universidades respondieron acertadamente. El quinto enunciado (Cuadro 1), se refiere a los cambios que puede producir la modificación genética en animales y la opción de respuesta correcta es "En desacuerdo". Para este enunciado, el porcentaje de acierto fue de $82 \%$ para todos los estudiantes interrogados. De manera general, los resultados obtenidos en esta sección indican que los estudiantes de la UCR fueron los que respondieron más acertadamente a las preguntas sobre actividades biotecnológicas, seguido por los estudiantes del ITCR y de la UNA respectivamente (Cuadro 1).

Las respuestas a los enunciados 1 a 5 analizados previamente, permitieron realizar una estimación del grado de conocimiento de la biotecnología tanto para los estudiantes de todas las universidades consideradas en conjunto, como para los de cada universidad por separado. De las 750 respuestas obtenidas, se observa en la figura 3, que el $88 \%$ de los encuestados respondieron correctamente de 3 a 5 de las afirmaciones y sólo un 12\% mostró un conocimiento insuficiente respecto al tema.

$\mathrm{Al}$ analizar las respuestas para los estudiantes de cada universidad (Fig. 4), se observa que un $81.6 \%$ de los estudiantes de la UCR parecen tener un buen conocimiento respecto a los temas biotecnológicos. El porcentaje es menor para los estudiantes del ITCR (65.6\%), y es más bajo para los encuestados de la UNA, donde un 54\% mostró un adecuado conocimiento de la materia (Fig. 4).

En relación con la tercera parte de la encuesta, una alta proporción de entrevistados $(83.8 \%)$, considera que las entidades encargadas de los aspectos regulatorios de las actividades biotecnológicas deben ser las "Organizaciones Científicas" (Cuadro 2). Le

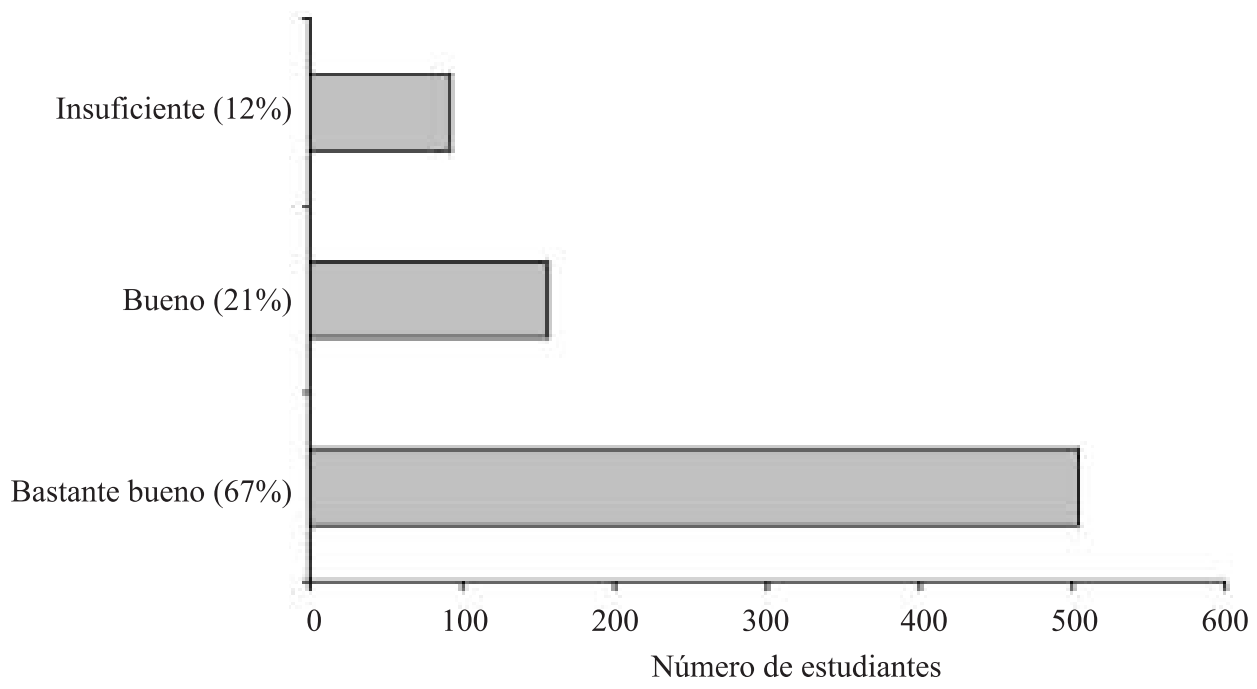

Fig. 3. Porcentaje (\%) de estudiantes universitarios de Costa Rica incluidos en este estudio, en acuerdo o en desacuerdo con los enunciados 1 a 5. Bastante bueno: 4 o 5 respuestas acertadas; Bueno: 3 respuestas acertadas; Insuficiente: menos de 3 acertadas.

Fig. 3. Percentage (\%) of Costa Rican university students included in this study, in agreement or not with statements 1 to 5. Very good: 4 or 5 correct responses; good: 3 correct responses; insufficient: less than 3 correct responses. 


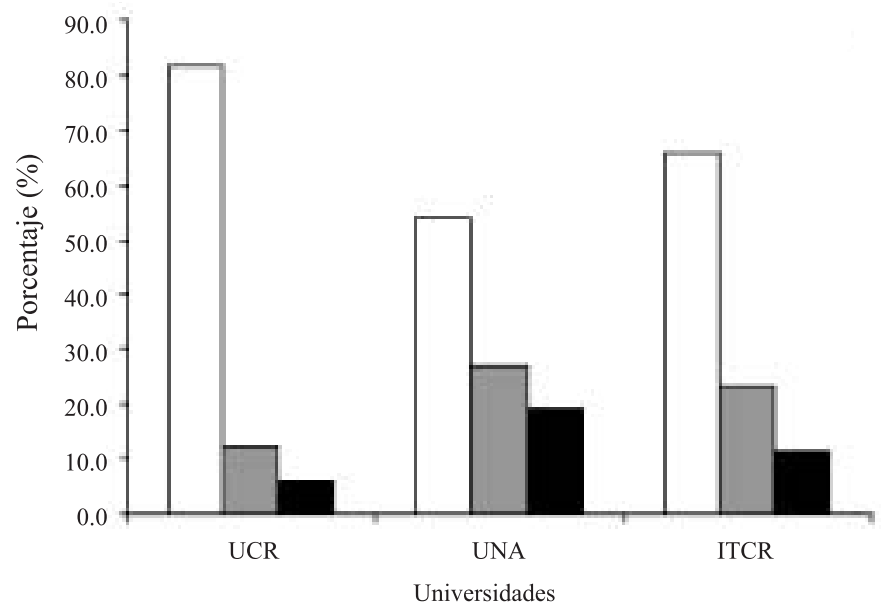

Fig. 4. Porcentaje (\%) de estudiantes de cada una de las universidades públicas de Costa Rica*, en acuerdo o en desacuerdo con los enunciados 1 a 5 (Cuadro 1). Bastante bueno: 4 o 5 respuestas acertadas; Bueno: 3 acertadas; Insuficiente: menos de 3 acertadas. Nota (*) UCR: Universidad de Costa Rica, UNA: Universidad Nacional, ITCR: Instituto Tecnológico de Costa Rica. $\square$ Bastante bueno $\square$ Bueno $\square$ Insuficiente.

Fig. 4. Percentage (\%) of students from each of the 3 public universities of Costa Rica*, in agreement or not with statements 1 to 5 (Table 1). Very good: 4 or 5 correct responses; good: 3 correct responses; insufficient: less than 3 correct responses. Note (*) Universidad de Costa Rica (UCR), Universidad Nacional (UNA) and Instituto Tecnológico de Costa Rica (ITCR). $\square$ Very good $\square$ Good $\square$ Bad..

\section{CUADRO 2}

Porcentaje (\%) de encuestados de las tres universidades públicas de Costa Rica consideradas en el estudio*, que respondieron afirmativa o negativamente en relación con las organizaciones que deberían regular las actividades biotecnológicas en el país

Organización

La Asamblea Legislativa

Comités de ética

Organizaciones Científicas

Organizaciones Internacionales

Organismos Públicos del Estado

22.8

* UCR: Universidad de Costa Rica; UNA: Universidad Nacional ; ITCR: Instituto Tecnológico de Costa Rica siguen en orden descendiente la opción de respuesta "Organizaciones Internacionales", "Comités de ética", "Organismos Públicos de Estado" y "La Asamblea Legislativa".

El Cuadro 3 presenta los resultados de la cuarta y última parte de la encuesta. Los resultados obtenidos en las respuestas a los tres enunciados presentados, fueron estadísticamente heterogéneos entre las tres universidades $\left(X^{2} \mathrm{p}<0.05\right)$. Por lo anterior, no se realizó un análisis promedio de los entrevistados para cada una de las preguntas de esta parte. En el enunciado 6: "Deben usarse solo métodos de cría tradicional en lugar de cambiar las características hereditarias de plantas y animales", la opción "En desacuerdo" denotaría una aceptación del uso de técnicas de mejoramiento genético moderno en agricultura y ganadería. Un 


\section{CUADRO 3}

Porcentaje (\%) de entrevistados de cada una de las universidades públicas de Costa Rica consideradas en el estudio*, que respondieron si estaban de acuerdo o en descuerdo con tres enunciados (6, 7 y 8) sobre biotecnología e ingeniería genética

\section{Enunciados}

6. Deben usarse solo métodos de cría tradicional en lugar de cambiar las características hereditarias de plantas y animales.

7. Los alimentos genéticamente modificados son peligrosos.

8. Se debe aceptar cierto grado de riesgo asociado a la biotecnología, si ésta refuerza la competitividad de Costa Rica.

\section{Respuesta \\ UCR}

Acuerdo

Desacuerdo

No responde

Acuerdo

Desacuerdo

No responde

Acuerdo

Desacuerdo

No responde

28

71.2

0.8

26.4

71.6

2

66

33.2

0.8

\section{UNA}

54.4

45.2

0.4

66

33.6

0.4

62

35.6

2.4
ITCR 48

51.6

0.4

50

49.6

0.4

68

31.2

0.8

* UCR: Universidad de Costa Rica; UNA: Universidad Nacional; ITCR: Instituto Tecnológico de Costa Rica.

$71.2 \%$ de los estudiantes de la UCR contestaron de manera favorable a esa práctica, mientras que un $51.6 \%$ de los estudiantes del ITCR y un $45.2 \%$ de los de la UNA, lo hicieron de manera favorable. El enunciado 7 indaga la percepción de los estudiantes sobre los alimentos derivados de OMGs. La opción "En desacuerdo" denotaría una posición favorable ante tal tipo de alimentos. La respuesta fue variable a nivel de las instituciones: los estudiantes de la UCR mostraron el porcentaje más alto de aceptación $(71.6 \%)$ en comparación con los valores obtenidos para los del ITCR (49.6\%) y los de la UNA con sólo $33.6 \%$ de opinión favorable. Las respuestas obtenidas para el enunciado 8 reflejan una aceptación moderada del riesgo asociado a la biotecnología, si ésta aumenta la competitividad de Costa Rica. Sólo para este enunciado, se observó un alto grado de semejanza en las respuestas de los estudiantes de las tres instituciones.

La estimación general del grado de aceptación de la biotecnología y la ingeniería genética muestra que el $79 \%$ de los encuestados respondieron favorablemente de 2 a 3 de los enunciados: un $54 \%$ con tres enunciados, y un $25 \%$ con dos. Un $21 \%$ de los entrevistados estuvo de acuerdo con uno o con ninguno de los enunciados (Fig. 5). Cuando se analizan estos

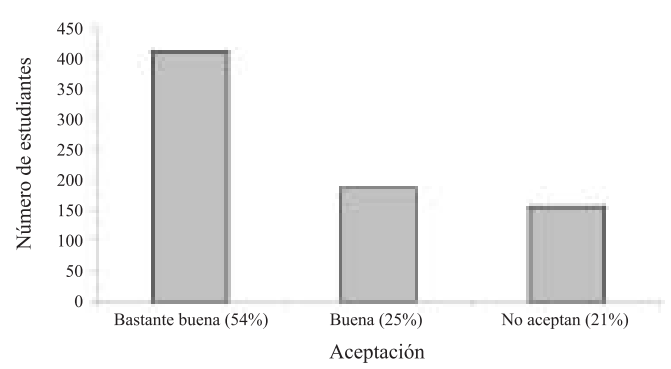

Fig. 5. Porcentaje (\%) de estudiantes de 3 universidades públicas de Costa Rica (UCR, UNA, ITCR), en acuerdo o en desacuerdo con tres enunciados (6, 7 y 8 del Cuadro 3$)$. Bastante buena: de acuerdo con 3 enunciados; Buena: de acuerdo con 2 enunciados. No aceptan: de acuerdo con 1 o con ninguno de los enunciados.

Fig 5. Percentage (\%) of students from 3 Costa Rican public universities (UCR, UNA, ITCR), in agreement or not with 3 statements (6, 7 and 8, Table 3). Very good: in agreement with 3 statements; good: in agreement with 2 statements; Do not accept: in agreement with one or none of the statements.

resultados para los estudiantes de cada universidad (Fig. 6), se observa una diferencia importante en las opiniones, que fue significativa $\left(X^{2} \mathrm{p}<0.05\right)$.

El Cuadro 4 presenta los porcentajes de estudiantes encuestados en cada área de estudio, para cada una de las tres universidades 


\section{CUADRO 4}

Porcentaje (\%) de estudiantes encuestados en las tres universidades públicas de Costa Rica*, según el área de estudio

$\begin{array}{lccc}\text { AREA DE ESTUDIO } & \text { UCR } & \text { UNA } & \text { ITCR } \\ \text { Artes y Letras } & 6 & 9 & - \\ \text { Ciencias Básicas } & 16 & 6 & 2 \\ \text { Ciencias Sociales } & 30 & 73 & 19 \\ \text { Ingenierías } & 29 & 11 & 79 \\ \text { Ciencias de la Salud } & 19 & 1 & -\end{array}$

* UCR: Universidad de Costa Rica; UNA: Universidad Nacional ; ITCR: Instituto Tecnológico de Costa Rica.

públicas de Costa Rica. La muestra de estudiantes de la UCR se encontró distribuida más homogéneamente entre las diferentes disciplinas de estudio, mientras que en las muestras de la UNA y del ITCR hubo mayor proporción de estudiantes del área de Ciencias Sociales, y de las Ingenierías, respectivamente. Cuando se comparó la proporción de estudiantes encuestados de cada universidad, de acuerdo a su área de estudio, con la distribución de estudiantes efectivamente matriculados en cada una de las disciplinas en el período de estudio (datos no presentados), se observó que la distribución de la muestra de la UCR fue representativa de la distribución de la población estudiantil matriculada en cada una de las áreas de estudio. La muestra de la UNA incluyó una alta proporción de estudiantes pertenecientes a las Ciencias Sociales, la cual efectivamente cuenta con un mayor número de estudiantes matriculados en estas disciplinas. De manera similar, en el ITCR la mayoría de estudiantes encuestados pertenecieron a las ingenierías (79.3\%), que es una de áreas que cuentan con mayor número de estudiantes matriculados.

\section{DISCUSIÓN}

Los resultados de esta investigación indican que en general, hay una buena percepción de las tecnologías como instrumentos para la

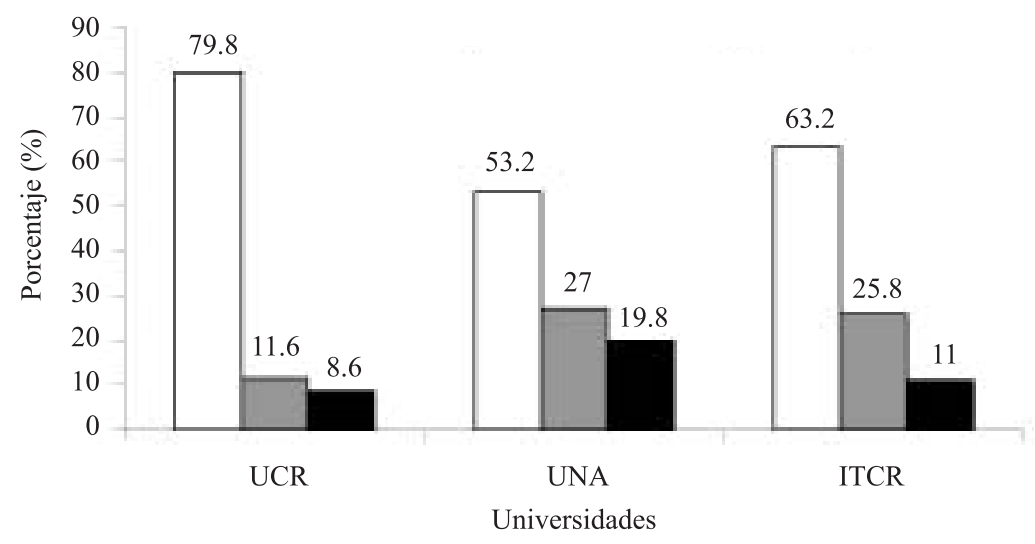

Fig. 6. Porcentaje (\%) de estudiantes de cada una de las 3 universidades públicas de Costa Rica consideradas en el estudio (UCR, UNA, ITCR), en acuerdo o en desacuerdo con 3 enunciados (6, 7 y 8 del Cuadro 3). Respuestas consideradas en conjunto para cada encuestado. Bastante buena: de acuerdo con 3 enunciados; Buena: de acuerdo con 2 enunciados. No aceptan: de acuerdo con 1 o con ninguno de los enunciados. $\square$ Bastante buena $\square$ Buena $\square$ No aceptan.

Fig 6. Percentage (\%) of students from each of the 3 public universities of Costa Rica considered in this study (UCR, UNA, ITCR), in agreement or not with 3 statements (6, 7 and 8, Table 3). Responses were considered together for each interviewed student. Very good: in agreement with 3 statements; good: in agreement with 2 statements; Do not accept: in agreement with 1 o none of the statements. $\square$ Very good $\square$ Good $\square$ Do not accept.. 
mejora en la calidad de vida en los próximos 20 años (Figs. 1 y 2), lo que es congruente con los resultados obtenidos en el Eurobarómetro sobre las actitudes generales a las nuevas tecnologías en los países europeos y Canadá (Gaskell et al. 2000, 2003).

La estimación del grado de conocimiento sobre algunas actividades y temas biotecnológicos mostró que los estudiantes tienen un grado satisfactorio, ya que la mayoría contestó en forma acertada a 4 o 5 de las preguntas (Fig. 3). Sólo $12 \%$ de los encuestados mostró un conocimiento insuficiente. En el mismo sentido, parecería que existe una opinión favorable (79\%), sobre el uso y los beneficios de la biotecnología, incluyendo los OMGs (Figs. 5 y 6).

Las diferencias de conocimiento y percepción pública de la biotecnología, encontradas entre las Universidades, podrían reflejar las diferencias en las áreas de estudio de los encuestados. Los estudiantes de ciencias sociales podrían tener un menor grado de conocimiento de temas relacionados con las ciencias de la vida, lo cual podría reflejarse en grados de actitud menos positivos hacia la biotecnología y la ingeniería genética. En ese sentido, podría considerarse que un mejor conocimiento de la materia, podría traducirse en un menor temor y consecuentemente, en un mayor nivel de aceptación de la biotecnología y de los OMGs, tal como lo sugieren otros estudios a nivel internacional (Fritz et al. 2003, Lang et al. 2003). En este contexto, se podría considerar que una mayor labor educativa sobre la biotecnología y sus aplicaciones, que se base en conocimientos y hechos científicos, y que se oriente hacia los diversos sectores de la sociedad y el público en general, tendría efecto en los niveles de percepción y de aceptación de estas tecnologías. Entonces, la comunidad científica y académica de Costa Rica, debería enfrentar este desafío, y prepararse para la innovación en el uso de los medios de comunicación, y para el fortalecimiento científico y tecnológico de los programas educativos del país.

Los entrevistados no parecen percibir el importante papel que deben tener las autoridades públicas (La Asamblea Legislativa y los organismos públicos del Estado) en el proceso de regulación de las actividades biotecnológicas. Podría deducirse que los entrevistados tampoco conocen las actividades regulatorias llevadas a cabo por el Comité Técnico Nacional de Bioseguridad, creado por ley del Estado en 1997 y adscrito al Ministerio de Agricultura y Ganadería. Este organismo es la autoridad que regula todas las actividades relacionadas con los OMGs, con la finalidad de garantizar la seguridad ambiental y de los alimentos derivados de cultivos genéticamente modificados en el país.

El uso de los cultivos transgénicos en la agricultura ha sido objeto de numerosos debates a nivel internacional (Gaskell et al. 2000, Priest 2000, Aerni 2001, Huete-Pérez y Orozco- González 2001), a menudo con una óptica estrictamente negativa, sin considerar las oportunidades que estos cultivos podrían brindar a los países en desarrollo. La adopción de cultivos transgénicos en otros países, tales como Estados Unidos, Canadá, China y Argentina, ha contribuido a la reducción en el uso de plaguicidas, así como a un abaratamiento de costos de producción, y a mayores beneficios para los agricultores (James 2003). Otros posibles beneficios para los países en desarrollo podrían ser la conservación de los bosques tropicales y de los recursos de la biodiversidad, o la generación de nuevos productos con características de alto valor agregado, tales como mejor calidad nutricional, resistencia o tolerancia a plagas y patógenos, retardo en la maduración de frutos, etc. (Trewavas 2001). En ese sentido, la comunidad científica y académica nacional, debe dar a conocer los beneficios y los riesgos potenciales asociados a las nuevas tecnologías. Así, el público podrá forjarse una opinión balanceada y objetiva sobre ellas. 


\section{AGRADECIMIENTOS}

Esta investigación fue financiada por la Vicerrectoría de Investigación de la Universidad de Costa Rica. Iris Rodríguez recibió apoyo del Servicio Alemán de Intercambio Académico (DAAD) para realizar estudios de posgrado en la Universidad de Costa Rica. Agradecemos a Juan Bautista Chavarría por su ayuda en el análisis estadístico de los resultados. A Elmer Guillermo García y a Oscar Rocha por sugerencias para el manuscrito.

\section{RESUMEN}

Se llevó a cabo una encuesta para estudiar la percepción y el grado de conocimiento sobre biotecnología y organismos modificados genéticamente (OMGs), en una muestra de estudiantes $(n=750)$ de tres universidades públicas de Costa Rica: Universidad de Costa Rica (UCR), Universidad Nacional (UNA) e Instituto Tecnológico de Costa Rica (ITCR). Se encontró que un $88 \%$ mostraron un conocimiento satisfactorio de la biotecnología moderna, y que un $79 \%$ expresaron una posición favorable y una buena aceptación de esa tecnología. Además, los estudiantes encuestados aceptarían ciertos riesgos asociados a la biotecnología, siempre y cuando, ésta mejore la capacidad competitiva de Costa Rica. El área de estudio de los estudiantes entrevistados parece estar relacionada con el grado de aceptación debido a que los estudiantes de disciplinas sociales mostraron una mayor percepción negativa hacia los productos biotecnológicos y OMGs, si se compara con la percepción expresada por los estudiantes encuestados de disciplinas de ciencias naturales y de áreas tecnológicas.

\section{REFERENCIAS}

Aerni, P. 2001. Assessing stakeholder attitudes to agricultural biotechnology in developing countries. Biotechnol. Dev. Monitor 47: 3-7.

Albertazzi, F.J. 2004. Scientific publications about DNA structure-function and PCR technique in Costa Rica: A historic view 1953-2003. Rev. Biol. Trop. 52: 13-17.

Cochran, W. G. 1963. Sampling techniques. Wiley. New York, USA. 413 p.
Durant, J. 2000. Eurobarometer, pp. 277-309. In Council of Europe (ed.). Proceedings of the international conference of the Council of Europe on ethical issues arising from the application of biotechnology. Oviedo, Spain, 16-19 May 1999. Council of Europe Publishing, Strasbourg Cedex.

Espinoza, A.M., A. Sittenfeld \& S. Salazar. 2003. Developing transgenic rice at the University of Costa Rica: perspectives and considerations for managing intellectual property rights. Interciencia 28 : 111-117.

Fritz, S., D. Husmann, G. Wingenbach, T. Rutherford, V. Egger \& P. Wadhwa. 2003. Awareness and acepptance of biotechnology issues among youth, undergraduates, and adults. AgBioForum 6: 178-184.

Garita-Bonilla, N. 2001. La Opinión pública como un espacio de diálogo, pp. 47-79. In M. Araya Incera (ed.). Estado de la opinión pública costarricense: un análisis de los principales temas de conflicto. CIMPA, San José, Costa Rica.

Gaskell, G., N. Allum, M. Bauer, J. Durant, A. Allansdottir, H. Bonfadelli, D. Boy, S. Cheveigné, B. Fjaestad, J. M. Gutteling, J. Hampel, E. JelsØe, J. Correia-Jesuino, M. Kohring, N. Kronberger, C. Midden, T.H. Nielsen, A. Przestalski, T. Rusanen, G. Sakellaris, H. Torgersen, T. Twardowsky \& W. Wagner. 2000. Biotechnology and the European public. Nat. Biotechnol. 18: 935-938.

Gaskell, G., N. Allum \& S. Stares. 2003. Europeans and biotechnology in 2002. Eurobarometer 58.0. A report to the EC Directorate General for Research from the project "Life Sciences in European Society" QLG7CT-1999-00286. 44 p.

Gómez-Barrantes, M. 1998. Elementos de estadística descriptiva. San José, Costa Rica. 564 p.

Huete-Pérez, J.A. \& D. Orozco-González. 2001. Percepciones sobre biotecnología en Nicaragua. ENCUENTRO 58: 83-90.

Lang, J. T., K.M. O’Neill \& W.K. Hallman. 2003. Expertise, trust and communication about food biotechnology. AgBioForum 6: 185-190.

Priest, S.H. 2000. US public opinion divided over biotechnology? Nat. Biotechnol. 18: 939-942.

Sasson, A. 1985. Les biotechnologies: defis et promesses. U.N.E.S.C.O, París, Francia. 336 p. 
Sittenfeld, A. \& A. M. Espinoza. 2002. Costa Rica: revealing data on public perception of GM crops. Trends Plant Sci. 7: 468-470.

Solleiro, J.L. \& R. Castañón. 2002. La Biotecnología en América Latina: panorama al año 2002. CamBioTec, Iniciativa Canadiense-Latinoamericana en Biotecnología para el Desarrollo Sustentable. IDRC, Canadá. 237 p.

Trewavas, A.J. 2001. The population/biodiversity paradox. Agricultural efficiency to save wilderness. Plant Physiol. 125: 174-179.

\section{REFERENCIAS DE INTERNET}

BIO (Biotechnology Industry Organization). 2002. BIO’s Editors' and Reporters' Guide to Biotechnology. 2002-2003. Consultado agosto2003, http: www.bio.org.

James, C. 2003. Preview-global starus of commercialized transgenic crops. (ISAAA Briefs No 30). Consultado febrero 2004, http: www.isaaa.org/CBT News/press_release/briefs 30/es_b30.pdf. 